\title{
Dermatologic manifestations of endocrine disorders
}

\author{
Michael Lause ${ }^{1}$, Alisha Kamboj ${ }^{1}$, Esteban Fernandez Faith ${ }^{1,2}$ \\ ${ }^{1}$ The Ohio State University College of Medicine, Columbus, OH, USA; ${ }^{2}$ Division of Dermatology, Department of Pediatrics, Nationwide Children's \\ Hospital, Columbus, OH, USA \\ Contributions: (I) Conception and design: All authors; (II) Administrative support: All authors; (III) Provision of study materials or patients: All \\ authors; (IV) Collection and assembly of data: All authors; (V) Data analysis and interpretation: All authors; (VI) Manuscript writing: All authors; (VII) \\ Final approval of manuscript: All authors. \\ Correspondence to: Esteban Fernandez Faith, MD. Division of Dermatology, Department of Pediatrics, Nationwide Children's Hospital, 700 Children's \\ Drive, Columbus, OH 43205, USA. Email: esteban.fernandezfaith@nationwidechildrens.org.
}

\begin{abstract}
The skin serves as a window for clinicians to understand, diagnose, and monitor endocrine disease. Dermatologic manifestations of endocrinopathies contribute significantly to an individual's health and quality of life. In this review, we outline various disorders of the hypothalamic-pituitary axis, thyroid gland, pancreas, adrenal gland, and androgen axis as well as hereditary endocrine syndromes. In acromegaly, glycosaminoglycan deposition contributes to a thickening of skin and soft tissue, which manifests as coarsening and enlargement of facial and acral structures. Stimulation of the thyrotropin receptor in hyperthyroidism results in mesenchymal tissue proliferation and consequent pretibial myxedema; other associated cutaneous features include onycholysis, and hyperhidrosis. Individuals with hypothyroidism exhibit cold, dry skin and brittle hair as well as a jaundice-like appearance due to carotene excess. The cutaneous features of diabetes mellitus (DM), mediated to a large extent by hyperglycemia and hyperinsulinemia, include necrobiosis lipoidica diabeticorum (NLD), diabetic dermopathy, and acanthosis nigricans. Pediatric patients with Cushing's syndrome almost invariably present with truncal obesity and growth retardation; disruption of collagen formation and the catabolic effects of hypercortisolism result in skin atrophy and purple abdominal striae. In patients with Addison's disease, generalized hyperpigmentation, secondary to elevated levels of melanocyte-stimulating hormone (MSH), is most prominent in sun-exposed areas. Due to hyperandrogenism, individuals with polycystic ovarian syndrome (PCOS) often exhibit hirsutism, acne vulgaris, and androgenetic alopecia. In multiple endocrine neoplasia (MEN) syndromes, specific gene mutations may lead to angiofibromas, lichen amyloidosis, and ganglioneuromas. Disruptions of immune regulation result in autoimmune polyglandular syndromes (APS) and associated clinical features including chronic mucocutaneous candidiasis, vitiligo, and alopecia areata. This paper highlights the underlying pathophysiology, dermatologic manifestations, and treatment of the aforementioned endocrine disorders.
\end{abstract}

Keywords: Dermatology; endocrine; cutaneous; manifestations

Submitted Aug 28, 2017. Accepted for publication Sep 20, 2017.

doi: $10.21037 /$ tp.2017.09.08

View this article at: http://dx.doi.org/10.21037/tp.2017.09.08

The endocrine and integumentary systems interact through a cohort of complex mechanisms. Dysregulation of endocrine hormones, as is seen in the many endocrinopathies, often results in clinically significant dermatologic disease. As these cutaneous lesions can serve as markers of endocrine disease and result in substantial morbidity, it is important for clinicians to be aware of their documented dermatologic manifestations.

\section{Disorder of the hypothalamic-pituitary axis}

\section{Acromegaly}

Growth hormone $(\mathrm{GH})$ excess generates an insidious cascade of clinical manifestations involving soft tissue and bone (1). This process is defined as gigantism or acromegaly based on whether it occurs prior to or following the fusion 
Table 1 Mucocutaneous manifestations of acromegaly

Macroglossia
Macrocheilia
Gingival hyperplasia
Coarse facial features
Cutis verticis gyrata
Hyperpigmentation
Acanthosis nigricans
Hyperhidrosis
Hypertrichosis
Nail changes

of the epiphyseal growth plates, respectively. Diagnosis of gigantism may occur at any age, and has been described as early as the second month of life (2). However, the incidence of gigantism is exceedingly rare, with one large study showing pituitary gigantism in only $0.6 \%$ of pediatric pituitary tumors (3). Acromegaly is most commonly diagnosed in adults, but features of this disorder may be present in adolescents with a GH excess disorder. More than $99 \%$ of cases of GH excess occur due to an adenoma of the pituitary gland (4). Occasionally, pancreatic and lung tumors produce a similar syndrome through ectopic production of GH releasing hormone (1). Diagnosis of this disorder typically begins with measurement of serum insulin-like growth factor 1 (IGF-1) (4). Following biochemical support of the diagnosis, magnetic resonance imaging aids in the identification and characterization of a pituitary tumor.

\section{Pathophysiology}

Hypersecretion of GH and IGF-1 is responsible for morphological changes in skin cells and adnexa. An increase in GH receptor stimulation causes proliferation of dermal fibroblasts while IGF-1 incites production of cultured keratinocytes. In sebaceous cells, GH promotes cell differentiation and IGF-1 increases DNA synthesis. Acromegalic skin thickening is attributed primarily to infiltration of the papillary dermis, reticular dermis, and sweat glands by glycosaminoglycan deposits. In individuals with acromegaly, these deposits are composed of hyaluronic acid (as in normal skin) as well as chondroitin-4 and 6-sulfate and dermatan sulfate, which contributes to their sizable and extremely absorbent nature (5).

\section{Dermatologic manifestations (Table 1)}

At the time of presentation, the vast majority of patients have some degree of cutaneous involvement, typically most conspicuous in the face, hands, and feet (6). Facial thickening and edema commonly manifests as coarsening of the chin, nose, and supraorbital ridges as well as prognathism, macroglossia, macrocheilia, and gingival hyperplasia. In addition to a pronounced nasolabial groove, increased size, redness, and wrinkling of the forehead can produce a melancholic expression. Cutis verticis gyrata and small fibromas due to fibrous tissue overgrowth are also common facial features (4). Cutis verticis gyrata is the result of an increase in dermal collagen and consequent buckling and coarse furrowing of skin at the posterior neck and on the scalp. In the hands and feet, acromegaly can cause a disproportionate enlargement of fingertips, leading to a drumstick-like appearance (1). Furthermore, alterations in skin pigment may present as diffuse hyperpigmentation in approximately $40 \%$ of patients. Less commonly, patients with acromegaly may develop acanthosis nigricans (4).

Involvement of skin appendages can also result in a variety of clinical effects. Increased production by sebaceous and sweat glands manifest as oily skin and hyperhidrosis, respectively (4). Moreover, involvement of the hair and nails can result in hypertrichosis as well as platonychia (flat, broad nail), respectively (7).

\section{Treatment}

Continuous treatment leads to significant reversal of softtissue and bone expansion due to a decline in GH levels (1). The current standard of treatment for acromegaly is transphenoidal excision of the pituitary adenoma followed by use of somatostatin analogs for residual disease. Adjuvant treatment options include dopamine agonists, such as bromocriptine, which suppress GH secretion. Studies have shown that 18-24 months of medical therapy are associated with complete reversal of most clinical features in $50 \%$ of patients; hyperhidrosis, however, persists in the majority of cases. In addition, refractory cases of cutis verticis gyrata may be treated with scalp reduction procedures for cosmetic purposes (8).

\section{Disorders of the thyroid gland}

\section{Hyperthyroidism}

\section{Introduction}

Hyperthyroidism is most commonly seen in Graves' 
Table 2 Cutaneous manifestations of hyperthyroidism

Warm, moist, smooth skin
Flushing
Palmar erythema
Hyperhidrosis
Diffuse scalp hair thinning
Onycholysis
Pretibial myxedema
Thyroid acropachy
Generalized pruritus
Chronic urticaria

disease with less common causes including thyroiditis, toxic multinodular goiter, toxic adenoma, and exogenous thyroid hormone use (4). The prevalence of Graves' disease in the United States is estimated to be over 3 million, with children accounting for at least 41,000 cases (9). Among the pediatric population, the peak incidence of Graves' disease occurs between 10 and 15 years of age and, like adults, it is more common among females (10). The first diagnostic step includes measurement of serum thyroid-stimulating hormone (TSH) and free thyroxine (FT4). If laboratory findings suggest hyperthyroidism, a radioactive iodine uptake and scan as well as testing for antibodies against the thyrotropin receptor may be conducted to discern the etiology.

\section{Pathophysiology}

Thyroid hormones potentiate both alpha- and betaadrenergic receptors in multiple tissues throughout the body. This manifests with various clinical responses including, but not limited to, tachycardia, increased cardiac output, increased body temperature, hyperhidrosis, and warm, moist skin. In Graves' disease, binding of the thyrotropin antibody to mesenchymal tissue receptors results in soft tissue proliferation and overgrowth (11).

\section{Dermatologic manifestations (Table 2)}

Common cutaneous manifestations of hyperthyroidism include flushing of the face, erythema of the palms, and hyperhidrosis of the palms and soles (4). The epidermis is found to be thin but not atrophic, such as one would observe in Cushing syndrome. Scalp hair often has a downy texture and diffuse scalp hair thinning is often noted, although the degree of hair loss has no correlation with disease severity. Nails are often described as soft and shiny. Onycholysis, or separation of the distal nail plate from the nail bed, is a common occurrence that can also be seen in hypothyroidism, psoriasis, allergic contact dermatitis, and trauma. The proximal portion of the nail plate remains a pink color while the distal portion changes to a white, opaque color. The resulting upturned nail plate, often referred to as a Plummer's nail, is most likely to be first seen on the fourth fingernail (1).

Pretibial myxedema, also known as thyroid dermopathy, is often associated with Graves' ophthalmopathy although it can also be seen in Hashimoto's thyroiditis. This phenomenon occurs in $4 \%$ of individuals with Graves' disease and is most commonly seen on the anterior tibia and dorsum of the feet. Physical exam findings include sharply demarcated pink or purple-brown papules or nodules overlying a non-pitting thickening and induration. The overlying skin may appear to be atrophic and transparent (4). Thyroid acropachy, a rare manifestation of Graves' disease (0.1-1\%) that is nearly always associated with ophthalmopathy and pretibial myxedema, is characterized by the triad of digital clubbing, acral swelling, and diaphyseal proliferation of the periosteum in acral and distal long bones. Thyroid acropachy is more common in adults, and only rarely reported in pediatric patients. This syndrome can be misdiagnosed as acromegaly, pulmonary osteoarthropathy, osteoperiostitis, and pachydermoperiostosis, but radiologic evidence of subperiosteal thickening can confirm the diagnosis of thyroid acropachy (12).

Less common cutaneous manifestations of hyperthyroidism include generalized pruritus, eczematous dermatitis, chronic urticaria and dermographism (4).

\section{Treatment}

Depending on the etiology of hyperthyroidism and patient characteristics, several treatment options may be employed to address the cutaneous manifestations by treating the underlying disease. Treatment options include antithyroid drugs, radioactive iodine ablation, and surgery. Beta adrenergic blockers have been shown to be effective in reducing moderate to severe symptoms of thyrotoxicosis. Hyperthyroidism secondary to thyroiditis tends to be selflimited in nature and can be managed with non-steroidal anti-inflammatory drugs in most cases.

When dermatologic manifestations persist, specific therapy may become necessary. For example, studies have 
Table 3 Cutaneous manifestations of hypothyroidism

Dry skin
Cold, mottled skin
Carotenemia
Myxedema
Macroglossia
Loss of lateral third of eyebrows
Coarse brittle hair

shown the efficacy of treating pretibial myxedema with intralesional corticosteroid injections; this treatment approach also applies to patients with similar findings secondary to hypothyroidism (13). Furthermore, treatments to combat hyperhidrosis include topical use of aluminum salts such as aluminum chloride and iontophoresis as firstline approaches. Systemic anticholinergic medications are often helpful when topical options fail. Botulinum toxin injections, liposuction, and sympathectomy may also be considered (14).

\section{Hypothyroidism}

\section{Introduction}

In the general population, the most common cause of hypothyroidism worldwide and in the United States is iodine deficiency and chronic lymphocytic thyroiditis (Hashimoto's thyroiditis), respectively (4). In the pediatric population, hypothyroidism may be congenital or acquired. Congenital hypothyroidism occurs in 1:2,000 to 1:4,000 newborns and is most often linked to thyroid agenesis. Studies have also identified a nearly 2:1 female to male predominance (15). Other common causes of hypothyroidism include radiationinduced hypothyroidism following ${ }^{131} \mathrm{I}$ treatment, postthyroidectomy, or drug therapy with lithium, bexarotene, or interferon (1). Hashimoto's thyroiditis in childhood has an estimated prevalence between one and two percent, with the majority of cases occurring among females (16). The signs and symptoms of hypothyroidism are non-specific and require biochemical support with the measurement of TSH and FT4. Subclinical hypothyroidism, which is described as an elevated TSH level with a normal FT4 level, may be seen in the early stages of hypothyroidism. The diagnosis of Hashimoto's thyroiditis can be confirmed through testing for anti-thyroid antibodies, which are present in $95 \%$ of patients (17).

\section{Pathophysiology}

In hypothyroidism, a reduced sensitivity of alpha- and betaadrenergic receptors to catecholamine signaling contributes to a lower basal metabolic rate and reduced response to sympathetic nervous system stimulation.

\section{Dermatologic manifestations (Table 3)}

Congenital hypothyroidism, a condition caused by thyroid hormone deficiency in fetal life, commonly demonstrates intellectual disability. In addition, these individuals may develop signs of myxedema, orbital hypertelorism, a protruding abdomen with associated umbilical hernia, acral swelling, a clavicular fat pad, and slow growing nails (18).

Individuals with acquired hypothyroidism often have cold, mottled, dry skin. It has been found that in over $80 \%$ of individuals with primary hypothyroidism, the epidermis is thin, rough, and hyperkeratotic giving a scaly appearance. Meanwhile, patients with hypothyroidism due to pituitary failure often demonstrate a fine wrinkling of the skin that imparts a parchment-like quality. In addition, excess carotene, due to reduced hepatic conversion of betacarotene to vitamin $\mathrm{A}$, can deposit in the stratum corneum and manifest as a yellowing of the skin. This process most commonly affects the palms, soles, and nasolabial folds, and a lack of scleral involvement can differentiate the clinical manifestations of carotenemia from jaundice (4).

In severe cases of hypothyroidism, accumulation of mucopolysaccharides (myxedema) can occur. The hands, face, pretibial, and periorbital areas are most commonly affected resulting in a non-pitting edema. The eyes can appear puffy or swollen, the outer third of the eyebrow can be lost, and ptosis can occur due to decreased sympathetic stimulation. Additional features of myxedema include a broadening of the nose, thickening of the lips, and macroglossia. Facial expressions may be blunted which can limit others' abilities to interpret emotion (4).

Individuals with hypothyroidism often have rough and brittle hair at least partly due to reduced sebum secretion. In addition, diffuse, partial alopecia, often including genital and beard hair, has been described in up to $50 \%$ of patients with hypothyroidism (4). Conversely, hypertrichosis of the back and shoulders can be a sign of hypothyroidism in the pediatric population (19). The nails are often described as brittle and striated. Lastly, other autoimmune conditions are observed at a greater frequency, including dermatitis herpetiformis, alopecia areata, vitiligo, and autoimmune urticaria (4). 
Table 4 Cutaneous manifestations of diabetes mellitus

Necrobiosis lipoidica diabeticorum (NLD)
Diabetic dermopathy
Diabetic bullae
Scleredema
Acanthosis nigricans
Carotenemia
Lipodystrophy
Hemochromatosis
Eruptive xanthomas
Cutaneous infections (bacterial, candidal, dermatophytes)

\section{Treatment}

The standard of treatment for individuals with hypothyroidism begins with supplementation with L-thyroxine which will reverse the skin findings of hypothyroidism. TSH levels are measured 6 weeks after starting L-thyroxine therapy or following a change in dosage. Dosage adjustments are made with the goal to normalize TSH levels and reduce the signs and symptoms of hypothyroidism.

\section{Disorder of the pancreas}

\section{Diabetes mellitus (DM)}

\section{Introduction}

DM is a group of disorders defined by hyperglycemia over an extended period of time. Insulin-dependent (type 1) and non-insulin dependent (type 2) diabetes are the most common subtypes. The estimated number of individuals, younger than 20 years of age, living with type 1 diabetes (T1DM) in the United States is approximately 200,000; an estimated $45 \%$ of children present before the age of 10. In addition, the increased prevalence of obesity and insulin resistance in the pediatric population today is associated with a higher incidence rate of type $2 \mathrm{DM}$ (T2DM) $(20,21)$.

\section{Pathophysiology}

Persistent hyperglycemia and hyperinsulinemia in T2DM lead to the protean manifestations of DM (1). Hyperglycemia results in non-enzymatic glycosylation of the vascular basement membrane with subsequent development of hyaline arteriolosclerosis. The resultant deteriorating microcirculation leads to peripheral vascular disease with a cohort of micro- and macro-vascular visceral complications. Hyperinsulinemia in T2DM leads to increased signaling through IGF-1 receptors, which are found in cultured fibroblasts and keratinocytes. These receptors bind IGF-1 with 100-1,000 times greater affinity than insulin. Thus, the insulin-dependent increase in IGF-1 and activation of the IGF-1 signaling pathway promotes cellular growth and differentiation and mediates the subsequent development of some of the cutaneous findings such as acanthosis nigricans (22).

\section{Dermatologic manifestations (Table 4)}

Patients with DM may present with different cutaneous manifestations, some of which are specific to DM, while others are non-specific or secondary to metabolic changes. Patients with DM have an increased susceptibility to cutaneous infections (i.e., staphylococcal pyodermas, candidiasis, erythrasma, and dermatophyte infections). At the same time, patients with T1DM have a tendency to develop autoimmune related cutaneous disorders (4).

Necrobiosis lipoidica diabeticorum (NLD) occurs in $0.3 \%$ to $1.6 \%$ of diabetic patients with a predisposition for affecting women and those who are insulin dependent (22). Approximately $75 \%$ of cases of NLD occur in patients with DM or those who will develop DM. In a minority of cases, NLD is seen without the association of DM. In pediatric patients, the diagnosis of NLD portends a greater risk of developing diabetic nephropathy and retinopathy (23). The lesions of NLD are well-demarcated oval or irregularly shaped, indurated plaques with yellow pigmentation at the center and red-brown or violaceous pigmentation at the periphery (Figure 1). Later in the course, the center may become atrophic with superficial telangiectasias (4). The lesions are most often found on the anterior or lateral lower leg and are found to ulcerate in up to $33 \%$ of patients (18).

Diabetic dermopathy is the most common dermatologic marker of DM, affecting up to $70 \%$ of adult patients with DM (24). Although it is commonly seen in adults, it can occasionally manifest in children as irregularly shaped, light brown macules and patches that are most commonly found on the anterior shin (18). Upon resolution of the lesions, patients may develop areas of atrophy or scarring. In addition, diabetic bullae are a rare ( $0.5 \%$ of patients) but specific finding in DM, and case reports have described this finding in the pediatric population. The large, non- 


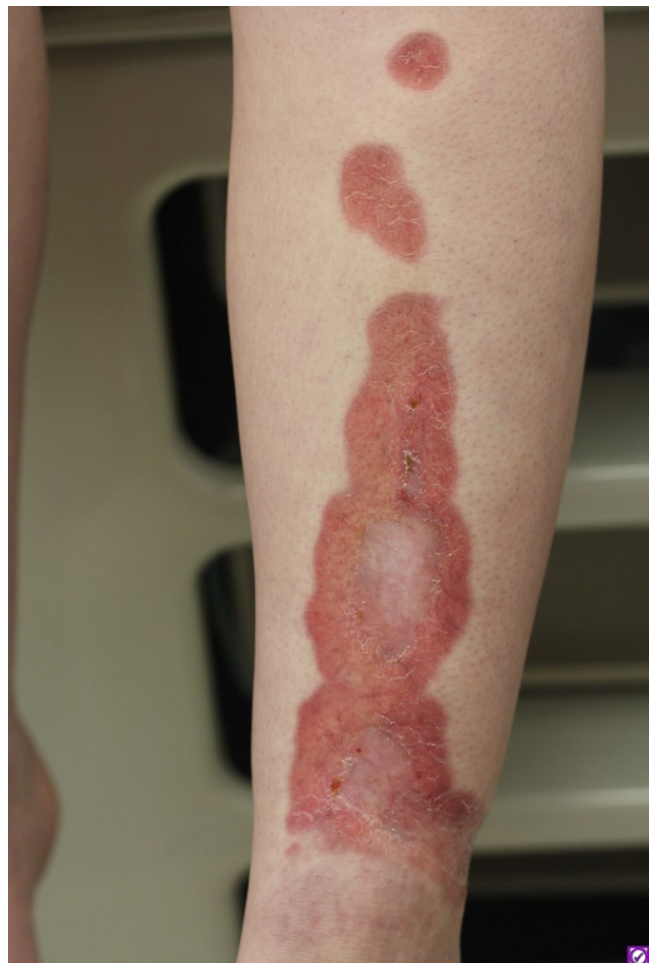

Figure 1 NLD. Yellow-brown plaques with central atrophy on the anterior leg. NLD, necrobiosis lipoidica diabeticorum.

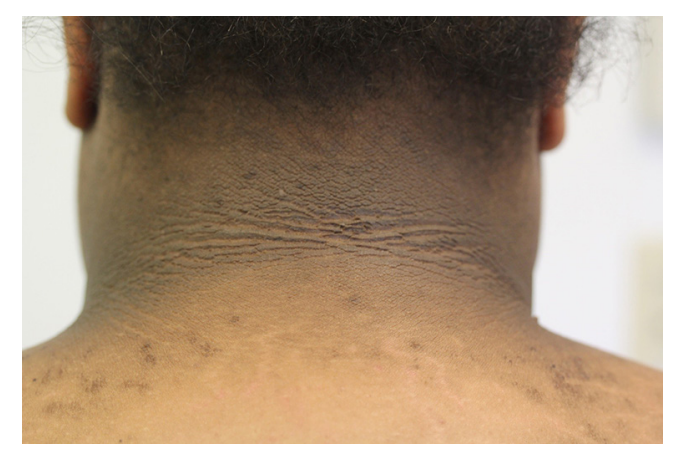

Figure 2 Acanthosis nigricans. Velvety, hyperpigmented plaque on the posterior and lateral neck.

inflammatory blisters usually occur sporadically and with no preceding trauma and are most likely to be found on the dorsolateral foot and ankle. The often asymptomatic bullae tend to be well-demarcated with no surrounding deformity and heal without scarring within 4 to 5 weeks (25).

Scleredema adultorum is also more common in adult patients with DM, and only rarely reported in children. It presents as painless, non-pitting swelling that starts on the lateral and posterior neck and may spread to the anterior neck, shoulders, face, and upper torso (26).

Several additional cutaneous disorders can be observed in patients with DM but are not specific to the disease. Acanthosis nigricans, described as a diffuse, velvety thickening and hyperpigmentation of the skin (Figure 2), has been associated with T2DM. It has a strong tendency to involve the axillae, neck, inframammary folds, inguinal folds, and perineum (4). Acanthosis nigricans occurs more frequently among children who are obese as well as those who are African or Native American (27).

Along with acanthosis nigricans, it is common to see skin tags or acrochordons particularly in overweight patients. Vitiligo occurs at a greater frequency in individuals with DM. As an autoimmune condition, patients with T1DM appear to be at higher risk (4). Other cutaneous manifestations in patients with DM include carotenemia, lipodystrophy, hemochromatosis, and xanthomatosis secondary to hyperlipidemia (4).

\section{Treatment}

The current standard of treatment for individuals with T1DM is daily subcutaneous insulin injections using a combination of long- and short-acting types. Individuals with T2DM are counseled on exercise and diet modifications. Medication options for T2DM include: metformin, secretagogues, thiazolidinediones, and $\alpha$-glucosidase inhibitors. If therapeutic goals are not met with the above agents, insulin may be added to the treatment regimen for patients with T2DM.

Treatments targeting specific cutaneous features of DM have also been studied. Both topical and intralesional corticosteroids have shown efficacy in treatment of NLD. Caution should be taken as corticosteroids can worsen the atrophic epidermis or ulcerated lesions often seen in NLD (18). In addition, pentoxifylline has shown promise in reducing ulceration, atrophy, and inflammation associated with NLD (28). Although no effective treatment exists for acanthosis nigricans, weight loss often leads to improved appearance of this finding. Retinoids (topical or systemic), and keratolytic agents (i.e., salicylic acid, lactic acid, urea) may also aid in managing acanthosis nigricans (18). In the treatment of scleredema, case reports have shown success with intravenous penicillin, low-dose methotrexate, and bath PUVA (1). Finally, management of diabetic dermopathy and diabetic bullae revolve around appropriate wound care to accelerate healing and prevent infection (25). 
Table 5 Cutaneous manifestations of Cushing syndrome

Moon facies

Dorsocervical fat pad

Supraclavicular fat pad

Easy bruising

Delayed wound healing

Skin atrophy

Striae distensae

Hyperpigmentation

Acanthosis nigricans

Steroid acne

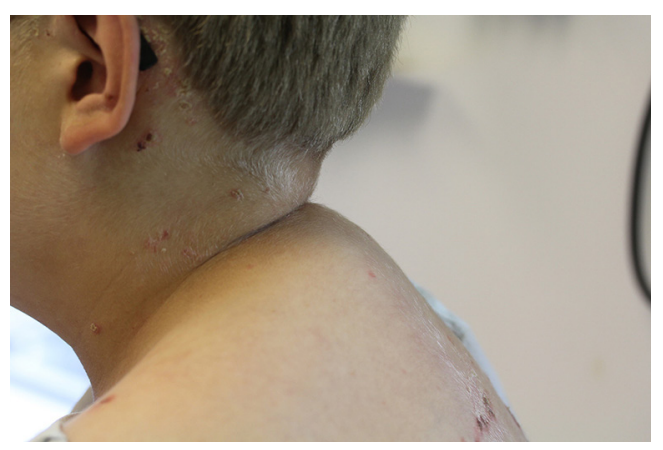

Figure 3 Dorsocervical fat pad: fat deposition on the posterior neck and upper back in a patient with iatrogenic Cushing syndrome.

\section{Disorders of the adrenal gland}

\section{Cushing syndrome}

Cushing syndrome is a collection of clinical features primarily caused by hypercortisolemia. The majority of cases of Cushing syndrome occurs due to pituitary (Cushing's disease) or ectopic hypersecretion of adrenocorticotropic hormone (ACTH), adrenal overproduction of cortisol, or iatrogenic administration of glucocorticoids (1). Although Cushing disease demonstrates a female predominance among adolescents and adults, studies show that pre-pubertal boys are affected more frequently than girls (29). The vast majority of children with Cushing syndrome present with growth retardation, which often triggers workup of this disorder. Further testing is indicated if two of the following screening tests are found to be positive: 24-hour urine free cortisol, overnight dexamethasone suppression test, and late-night salivary cortisol. Measurement of ACTH can subsequently characterize the pathologic source while imaging can aid in considering interventions (4).

\section{Pathophysiology}

The mechanisms highlighting the therapeutic and side effect profiles of glucocorticoids are varied and only partially understood. Excess levels of glucocorticoids are thought to decrease proliferation of keratinocytes and dermal fibroblasts. These changes abate the formation of collagen and other components of the extracellular matrix and ultimately contribute to skin atrophy and fragility. The catabolic effects of glucocorticoids extend to subcutaneous connective tissue as well (30).

\section{Dermatologic manifestations (Table 5)}

Pediatric patients with Cushing syndrome almost invariably present with truncal obesity and retardation of growth. In contrast to growth charts for children with exogenous obesity, which reveal increasing linear growth, growth charts for children with glucocorticoid excess typically demonstrate diminished linear growth with concurrent weight gain across percentiles. Deposition of adipose tissue in the following characteristic locations contributes to the Cushingoid appearance: cheeks ('moon facies'), dorsocervical fat pad ('buffalo hump', Figure 3), supraclavicular fat pad (thick, short neck), and behind the orbit (exophthalmos). Furthermore, loss of subcutaneous tissue can cause easy bruising and delayed wound healing (4).

Cushing syndrome is also accompanied by numerous distinct cutaneous changes. 'Cigarette paper' skin on the elbows and dorsum of the hands is the result of epidermal and dermal atrophy. When severe, application of adhesive tape causes peeling of this delicate skin. As the body's mass redistributes and enlarges, the fragile skin stretches and subcutaneous blood vessels become more easily visible, appearing as purple striae. The violaceous, thick $(>1 \mathrm{~cm}$ in diameter) striae of Cushing syndrome can be distinguished from the pink-silver, thin striae observed in growth spurts, obesity, and pregnancy (4). These physiologic striae may be seen in up to $35 \%$ of girls and $15 \%$ of boys between 9-16 years of age (18). Additional changes in the appearance of skin may manifest as acanthosis nigricans due to metabolic changes or hyperpigmentation in ACTHdependent forms (4). Excess endogenous or exogenous glucocorticoids may also lead to steroid acne. This presents as erythematous, monomorphic papules or small 


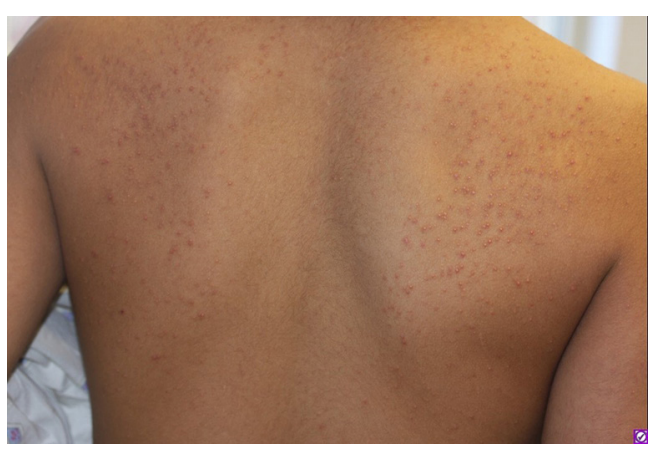

Figure 4 Steroid-induced acne. Monomorphic erythematous papulo-pustules on posterior trunk caused by exogenous use of corticosteroids.

Table 6 Mucocutaneous manifestations of Addison's disease

Diffuse cutaneous hyperpigmentation

Hair hyperpigmentation

Mucosal hyperpigmentation

Loss of axillary and pubic hair

Longitudinal hyperpigmented nail bands

pustules distributed along the upper trunk, proximal upper extremities, neck and face (Figure 4) (30). Glucocorticoid excess can also predispose individuals to superficial dermatophyte and pityrosporon infections (1).

\section{Treatment}

Other than striae, the majority of dermatologic manifestations associated with Cushing syndrome resolve after therapy targeted at excess cortisol production. For localized tumors, surgery is often employed. Treatment regimens may also utilize chemotherapy, radiation, and steroid synthesis inhibitors. Although management of striae generally yields unsatisfactory results, many of the striae that manifest in adolescence become less noticeable over time. In addition, some reports suggest the efficacy of topical tretinoin cream, acid peels, and topical hyaluronic acid preparations. Other treatment modalities include laser therapies, intense pulsed light, and microdermabrasion (31).

\section{Addison's disease/primary adrenal insufficiency}

Primary adrenal insufficiency is mediated most commonly by autoimmune mechanisms and is commonly referred to as Addison's disease. Among children, it represents 15\% of cases of primary adrenal insufficiency (32). Addison's disease can exist as an isolated condition or as a component of the autoimmune polyglandular syndromes (APS) (types 1 and 2). Workup of this disorder entails demonstration of inappropriately low cortisol secretion, determination of ACTH status to distinguish primary versus secondary adrenal insufficiency, and imaging of the adrenal glands (1). Common laboratory derangements in Addison's disease include hyponatremia, hyperkalemia, and hyperchloremic metabolic acidosis.

\section{Pathophysiology}

Decreased cortisol production from the damaged adrenal glands results in a loss of negative feedback on the hypothalamic pituitary axis. This increases the production of corticotropin releasing hormone and thereby, proopiomelanocortin, a precursor to the biologically active hormones ACTH, melanocyte-stimulating hormone (MSH), and others. Elevated levels of MSH consequently trigger melanin synthesis by epidermal melanocytes (33).

\section{Dermatologic manifestations (Table 6)}

Generalized bronze hyperpigmentation, the result of increased melanin production, is observed in almost all adult patients and $67 \%$ of pediatric patients. It is most prominent in sun-exposed areas as well as areas of trauma and pressure. Other frequently affected body parts include the axilla, areolas, perineum, and palmar creases. In some children, pigmentation may be quite diffuse and comparison of children to other family members may be useful in ascertaining clinical findings. Following the onset of Addison's disease, individuals may develop hyperpigmented scars, darkening of hair, and longitudinal bands on nails. Irregular or streaked, blue-black or brown hyperpigmentation of mucosal surfaces may also ensue and most commonly involves the tongue, inner surface of the lip, as well as buccal and gingival mucosa. In addition, patients may notice darkening of existing nevi (34). Vitiligo, caused by the autoimmune destruction of dermal melanocytes, occurs in $10-20 \%$ of individuals with autoimmune adrenalitis (4). In these patients, the concurrent presence of Addison's disease and vitiligo may give patients a striking mixture of pigment loss and hyperpigmentation. Aside from changes in pigment, women with Addison's disease may experience a loss of axillary and pubic hair, which are dependent on adrenal androgen production (4). 
Table 7 Cutaneous manifestations of androgen excess

Hirsutism

Acne vulgaris

Androgenetic alopecia

Acanthosis nigricans

\section{Treatment}

The treatment of Addison's disease involves replacement of glucocorticoids and mineralocorticoids that the adrenal gland is unable to produce. The dosage of glucocorticoids is adjusted based on clinical context.

\section{Disorder of androgen excess}

\section{Polycystic ovarian syndrome (PCOS)}

The most common disorder associated with androgen excess is PCOS; other common causes include congenital adrenal hyperplasia as well as ovarian and adrenal tumors. PCOS is characterized by evidence of hyperandrogenism and chronic anovulation in the absence of pituitary or adrenal pathology (4). Among adolescents, cases of PCOS usually come to medical attention due to complaints of menstrual irregularity (18). However, signs of androgen excess, such as severe acne or hirsutism, may also trigger an evaluation. Initial workup for a suspected androgenrelated disorder typically entails measurement of serum dehydroepiandrosterone-sulfate (DHEA-S), testosterone, and prolactin (4).

\section{Pathophysiology}

Although the pathophysiology of PCOS has not been entirely elucidated, several hormonal pathways likely contribute to its associated reproductive and metabolic sequelae. Individuals with PCOS demonstrate increased pulsatile action of gonadotropin-releasing hormone, leading to preferential secretion of luteinizing hormone (LH). Elevations in $\mathrm{LH}$ and insulin contribute to the hallmark androgen excess observed in this syndrome.

\section{Dermatologic manifestations (Table 7)}

PCOS is linked to several cutaneous features. It is the most common cause of hirsutism, which is defined as excess terminal hair growth in a male-pattern distribution including the beard area (face and neck), back, chest and lower abdomen (35). Hyperandrogenism contributes to this clinical presentation by increasing the circumference of hair fibers and prolonging the anagen phase of hair growth in the beard, axillary, and pubic regions. Androgen levels do not, however, correlate directly with the quantity of hair growth. Acne vulgaris is an additional manifestation of PCOS (36). Consideration for androgen excess should be given to patients with moderate to severe acne, particularly patients whose acne appears to be resistant to conventional therapies or recurrence after treatment with isotretinoin. Virilization can be another consequence of androgen excess and is most often characterized by deepening of the voice, muscle hypertrophy, reduction in breast size, clitoromegaly, and androgenetic alopecia (4). Individuals with hair loss often notice a gradual thinning of the hair that most commonly affects the vertex and upper biparietal region of the scalp with preservation of the anterior hair line (37). Other common findings in this disorder include acanthosis nigricans-due to insulin resistance and hyperinsulinism-as well as seborrheic dermatitis (1).

\section{Treatment}

Initial management of PCOS includes weight loss and dietary modifications. Pharmaceutical approaches typically utilize oral contraceptives as a first-line measure with the addition of metformin for insulin resistance and hyperglycemia as needed. For treatment of hirsutism and acne, anti-androgen therapy with an agent such as spironolactone may be effective. Additional treatment for hirsutism includes laser hair removal, electrolysis, and topical eflornithine (38). Treatment options for androgenetic alopecia in females include topical minoxidil and spironolactone.

\section{Hereditary endocrine syndromes}

\section{Multiple endocrine neoplasia (MEN) syndromes}

MEN syndromes represent a spectrum of autosomal dominant disorders that include benign and malignant neoplasms of multiple endocrine tissues. Variable endocrine involvement leads to a diverse range of clinical presentations, with cutaneous features often contributing to the identification of these disorders (18). MEN1 has an estimated prevalence of 1 to 10 per 100,000 individuals and is characterized by tumors of the parathyroid glands, endocrine pancreas, and anterior pituitary (39). The combined prevalence of MEN2A and 
MEN2B is approximately 1 per 35,000 individuals, with MEN2A representing $90 \%$ to $95 \%$ of cases (40). Common features of both MEN2 syndromes include medullary thyroid carcinoma and pheochromocytoma. MEN2A is distinguished by parathyroid hyperplasia while clinical findings of MEN2B include ganglioneuromatosis, and Marfanoid habitus.

\section{Pathophysiology}

Absence of a gene that encodes the tumor suppressor protein called Menin is responsible for the clinical constellation seen in MEN1. The features of both MEN2A and MEN2B occur due to a gain-in-function mutation in the RET protooncogene (18).

\section{Dermatologic manifestations}

Angiofibromas are the most common cutaneous manifestation of MEN1, with one study finding facial angiofibromas in $88 \%$ of individuals. These lesions appear as flesh-colored, pink, or red papules with a smooth and shiny surface (41). Other common dermatologic findings include lipomas, café-au-lait spots, and collagenomas (1). Although only occurring in 3\% of patients with MEN1, glucagonoma is associated with a distinct cutaneous manifestation, termed necrolytic migratory erythema (NME) (42). Often seen alongside new-onset DM, anemia, and stomatitis, NME initially presents as erythema of the groin and perineum and can eventually spread to the extremities and perioral region. The areas of erythema often progress to vesicles and bullae before a final crusting stage (43).

The most significant dermatologic manifestation of MEN2A is lichen amyloidosis. This condition has a predilection for the extensor surfaces of the upper back and extremities, with pruritus often the initial feature. Lesions appear as gray-to-brown, hyperkeratotic papules that have the potential to develop into larger plaques (44).

In MEN2B, mucosal neuromas are often seen by the fifth year of life and most commonly occur on the lips, tongue, eyelids, and conjunctiva. On the lips, ganglioneuromas can cause a diffuse, fleshy projection. The anterior one-third of the tongue is most likely to be involved and demonstrates clear-to-pink papules. Signs of eyelid or conjunctival involvement include a thickened eyelid margin as well as disorganized eyelashes. Lastly, patients with MEN2B are found to have café-au-lait macules at an increased incidence (45).

\section{Treatment}

The medical treatment of MEN syndromes is complex and each case requires individual attention. For patients with benign skin tumors (angiofibromas, lipomas, and collagenomas), surgical excision can be employed. In addition, topical rapamycin and laser therapy, used alone or concomitantly, can reduce the size and number of angiofibromas (46). There is no reported definitive therapy for lichen amyloidosis; however, a variety of case reports have found success with multiple treatment approaches including, but not limited to retinoids, corticosteroids, laser therapy, and phototherapy (47).

\section{APS}

APS are a heterogeneous group of immune-mediated disorders characterized by insufficiency of at least two endocrine organs (18). APS type I (APS I) is an autosomal recessive condition that most commonly presents in childhood, between 3 and 5 years of age, or in early adolescence. It is defined by the coexistence of 2 of 3 features: chronic mucocutaneous candidiasis, acquired hypoparathyroidism, and adrenal insufficiency (Addison's disease) $(48,49)$. Conversely, APS type II (APS II) displays a polygenic, autosomal dominant mode of inheritance and typically manifests in the third or fourth decade of life; it is the most common APS. This disorder is characterized by adrenal insufficiency in conjunction with autoimmune thyroid disease and/or type $1 \mathrm{DM}$ (48). Clinically, APS type III (APS III) excludes adrenal disease but is otherwise very similar to APS type II (18).

\section{Pathophysiology}

APS I is caused by a mutation in the AIRE gene, which is located on chromosome 21 and is responsible for the production of the "autoimmune regulator" protein (18). The pathophysiology of APS II is multifactorial, with both hereditary and environmental factors playing a role. Some of the component disorders associated with this syndrome have been linked to the class II human leukocyte antigen haplotypes DR3 and DR4 (49).

\section{Dermatologic manifestations}

Chronic mucocutaneous candidiasis is observed in the majority of patients with APS I and typically presents prior to the associated endocrinologic features (48). It refers to the presence of frequent or persistent fungal infections of the mucous membranes, skin, and nails. Oral candidiasis 


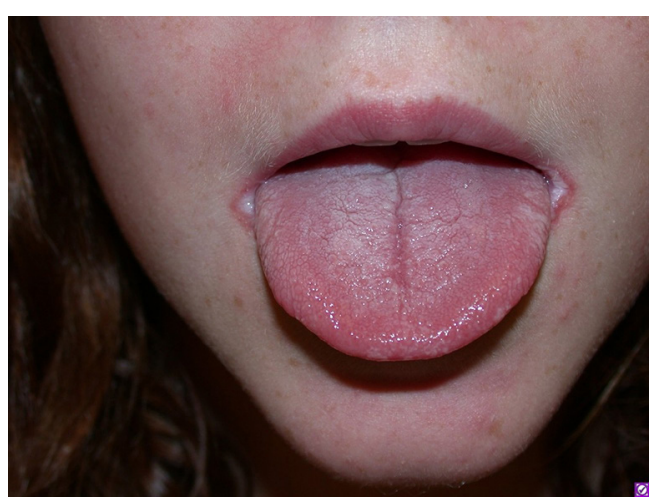

Figure 5 Angular cheilitis secondary to Candida albicans in a patient with APS. APS, autoimmune polyglandular syndromes.

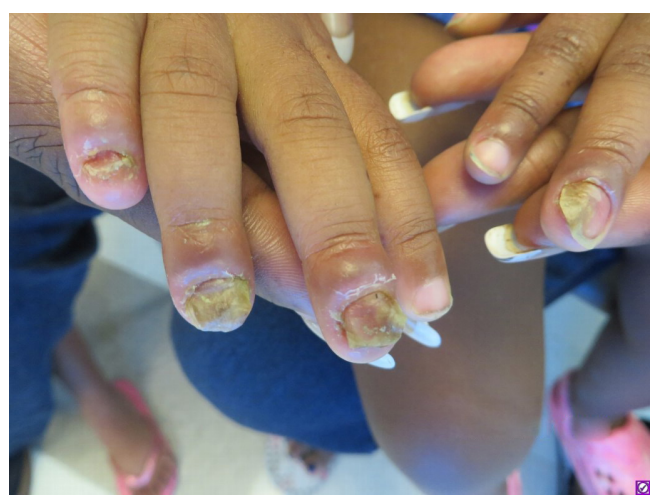

Figure 6 Chronic candidal paronychia: erythema and swelling of proximal nail folds along with yellowish thickening of nails.

peaks in incidence within the first 2 years of life and tends to follow a chronic course. Cutaneous candidal infections may result in angular cheilitis (Figure 5), scalp infection, intertrigo, and diaper or perianal candidiasis (50). Lastly, fungal infection of the nails leads to discoloration and thickening as well as paronychia (inflammation of the periungual skin, Figure 6) $(18,51)$.

Other dermatologic associations with APS I include alopecia areata and vitiligo, which affect approximately $30 \%$ and $<10 \%$ of patients, respectively $(18,52)$. Patients with APS II and III may also present with vitiligo and alopecia areata, albeit less frequently (51).

The clinical presentation of patients with APS I may be impacted by the presence of hypoparathyroidism as well. In this condition, the skin is typically dry, scaly, and hyperkeratotic. In addition, observation may reveal opaque, brittle nails with longitudinal grooves as well as sparse hair (4).

\section{Treatment}

In addition to management of endocrine abnormalities, treatment of the cutaneous features of APS employs the use of antifungal agents. Candidal infections typically respond well to azole medications. However, amphotericin may be required to treat severe cases $(53,54)$. The role of immunosuppressive therapy in managing APS is not currently well understood.

\section{Acknowledgements}

The authors would like to acknowledge Patricia Witman and Joy Mosser-Goldfarb for their contributions with clinical photographs.

\section{Footnote}

Conflicts of Interest: The authors have no conflicts of interest to declare.

\section{References}

1. Rustin MH. Andrews' diseases of the skin - clinical dermatology. Postgrad Med J 1990;66:984.

2. Abe T, Tara LA, Lüdecke DK. Growth hormonesecreting pituitary adenomas in childhood and adolescence: features and results of transnasal surgery. Neurosurgery 1999;45:1-10.

3. Rostomyan L, Daly AF, Petrossians P, et al. Clinical and genetic characterization of pituitary gigantism: an international collaborative study in 208 patients. Endocr Relat Cancer 2015;22:745-57.

4. Jabbour SA. Cutaneous manifestations of endocrine disorders: a guide for dermatologists. Am J Clin Dermatol 2003;4:315-31.

5. Ben-Shlomo A, Melmed S. Skin manifestations in acromegaly. Clin Dermatol 2006;24:256-9. Review.

6. Molitch ME. Clinical manifestations of acromegaly. Endocrinol Metab Clin North Am 1992;21:597-614.

7. Zayed A, Sobhi RM, Abdel Halim DM. Using trichloroacetic acid in the treatment of acanthosis nigricans: a pilot study. J Dermatolog Treat 2014;25:223-5.

8. Mishra A, Tehrani H, Hancock K, et al. Management of primary cutis verticis gyrata with tissue expansion and hairline lowering foreheadplasty. J Plast Reconstr Aesthet Surg 2010;63:1060-1. 
9. Jacobson DL, Gange SJ, Rose NR, et al. Epidemiology and estimated population burden of selected autoimmune diseases in the United States. Clin Immunol Immunopathol 1997;84:223-43.

10. Bansal S, Umpaichitra V, Desai N, et al. Pediatric Graves' disease. Int J Endocrinol 2015;1.

11. Bilezikian JP, Loeb JN. The influence of hyperthyroidism and hypothyroidism on alpha- and beta-adrenergic receptor systems and adrenergic responsiveness. Endocr Rev 1983;4:378-88.

12. Fatourechi V, Ahmed DD, Schwartz KM. Thyroid acropachy: report of 40 patients treated at a single institution in a 26-year period. J Clin Endocrinol Metab 2002;87:5435-41.

13. Lan C, Li C, Chen W, et al. A Randomized Controlled Trial of Intralesional Glucocorticoid for Treating Pretibial Myxedema. J Clin Med Res 2015;7:862-72.

14. Pride HB, Yan AC, Zaenglein A. Pediatric dermatology. 1st edition. Edinburgh; New York: Saunders Limited, 2008.

15. Rastogi MV, LaFranchi SH. Congenital hypothyroidism. Orphanet J Rare Dis 2010;5:17.

16. Hanley P, Lord K, Bauer AJ. Thyroid Disorders in Children and Adolescents: A Review. JAMA Pediatr 2016;170:1008-19.

17. Stevens CD, Miller LE. Clinical immunology and serology: A laboratory perspective. 4th edition. Philadelphia: F.A. Davis Company, 2017.

18. Mancini AJ, Goldsmith L, Paller A. Hurwitz clinical pediatric dermatology: A textbook of skin disorders of childhood and adolescence. Elsevier Inc., 2016.

19. Keen MA, Hassan I, Bhat MH. A clinical study of the cutaneous manifestations of hypothyroidism in kashmir valley. Indian J Dermatol 2013;58:326.

20. Writing Group for the SEARCH for Diabetes in Youth Study Group, Dabelea D, Bell RA, et al. Incidence of diabetes in youth in the United States. JAMA 2007;297:2716-24.

21. 2014 Statistics Report. Center for Disease Control and Prevention. Available online: https://www.cdc.gov/ diabetes/pdfs/library/diabetesreportcard2014.pdf

22. Van Hattem S, Bootsma AH, Thio HB. Skin manifestations of diabetes. Cleve Clin J Med 2008;75:772, 774, 776-7 passim.

23. Verrotti A, Chiarelli F, Amerio P, et al. Necrobiosis lipoidica diabeticorum in children and adolescents: a clue for underlying renal and retinal disease. Pediatr Dermatol 1995;12:220-3.

24. Brzezinski P, Chiriac AE, Pinteala T, et al. Diabetic dermopathy ("shin spots") and diabetic bullae ("bullosis diabeticorum") at the same patient. Pak J Med Sci 2015;31:1275-6.

25. Sibbald RG, Landolt SJ, Toth D. Skin and diabetes. Endocrinol Metab Clin North Am 1996;25:463-72.

26. Meguerditchian C, Jacquet P, Béliard S, et al. Scleredema adultorum of Buschke: an under recognized skin complication of diabetes. Diabetes Metab 2006;32:481-4.

27. Stuart CA, Gilkison CR, Smith MM, et al. Acanthosis nigricans as a risk factor for non-insulin dependent diabetes mellitus. Clin Pediatr (Phila) 1998;37:73-9.

28. Wee E, Kelly R. Pentoxifylline: An effective therapy for necrobiosis lipoidica. Australas J Dermatol 2017;58:65-8.

29. Storr HL, Isidori AM, Monson JP, et al. Prepubertal Cushing's disease is more common in males, but there is no increase in severity at diagnosis. J Clin Endocrinol Metab 2004;89:3818-20.

30. Schäcke H, Döcke WD, Asadullah K. Mechanisms involved in the side effects of glucocorticoids. Pharmacol Ther 2002;96:23-43.

31. Ud-Din S, McGeorge D, Bayat A. Topical management of striae distensae (stretch marks): prevention and therapy of striae rubrae and albae. J Eur Acad Dermatol Venereol 2016;30:211-22.

32. Perry R, Kecha O, Paquette J, et al. Primary adrenal insufficiency in children: twenty years experience at the Sainte-Justine Hospital, Montreal. J Clin Endocrinol Metab 2005;90:3243-50.

33. Erickson QL, Faleski EJ, Koops MK, et al. Addison's disease: the potentially life-threatening tan. Cutis 2000;66:72-4.

34. Sünkel S, Wichmann-Hesse A, Gärtner R, et al. Increasing pigmentation in Schmidt syndrome (polyglandular autoimmune syndrome type II. Hautarzt 2001;52:974-6.

35. Blank SK, Helm KD, McCartney CR, et al. Polycystic ovary syndrome in adolescence. Ann N Y Acad Sci 2008;1135:76-84.

36. Yildiz BO. Diagnosis of hyperandrogenism: clinical criteria. Best Pract Res Clin Endocrinol Metab 2006;20:167-76.

37. Herskovitz I, Tosti A. Female pattern hair loss. Int J Endocrinol Metab 2013;11.

38. Smith SR, Piacquadio DJ, Beger B, et al. Eflornithine cream combined with laser therapy in the management of unwanted facial hair growth in women: a randomized trial. Dermatol Surg 2006;32:1237-43.

39. de Laat JM, van der Luijt RB, Pieterman CR, et al. MEN1 redefined, a clinical comparison of mutation-positive and 
mutation-negative patients. BMC Med 2016;14:182.

40. Marquard J, Eng C. Multiple Endocrine Neoplasia Type 2. In: Adam MP, Ardinger HH, Pagon RA, et al. editors. GeneReviews®. Seattle (WA): University of Washington, Seattle; 1993-2017.

41. Darling TN, Skarulis MC, Steinberg SM, et al. Multiple facial angiofibromas and collagenomas in patients with multiple endocrine neoplasia type 1. Arch Dermatol 1997;133:853-7.

42. Giusti F, Marini F, Brandi ML. Multiple Endocrine Neoplasia Type 1. In: Adam MP, Ardinger HH, Pagon RA, et al. editors. GeneReviews ${ }^{\circledR}$. Seattle (WA): University of Washington, Seattle; 1993-2017.

43. Chastain MA. The glucagonoma syndrome: a review of its features and discussion of new perspectives. Am J Med Sci 2001;321:306-20.

44. Donovan DT, Levy ML, Furst EJ, et al. Familial cutaneous lichen amyloidosis in association with multiple endocrine neoplasia type 2A: a new variant. Henry Ford Hosp Med J 1989;37:147-50.

45. Jacobs JM, Hawes MJ. From eyelid bumps to thyroid lumps: report of a MEN type IIb family and review of the literature. Ophthal Plast Reconstr Surg 2001;17:195-201.

46. Madke B. Topical rapamycin (sirolimus) for facial angiofibromas. Indian Dermatol Online J 2013;4:54-7.

47. Vasani RJ. Response to oral acitretin in lichen amyloidosis. Indian Dermatol Online J 2014;5:S92-4.

48. Dittmar M, Kahaly GJ. Polyglandular autoimmune

Cite this article as: Lause M, Kamboj A, Fernandez Faith E. Dermatologic manifestations of endocrine disorders. Transl Pediatr 2017;6(4):300-312. doi: 10.21037/tp.2017.09.08 syndromes: immunogenetics and long-term follow-up. J Clin Endocrinol Metab 2003;88:2983-92.

49. Majeroni BA, Patel P. Autoimmune polyglandular syndrome, type II. Am Fam Physician 2007;75:667-70.

50. Perheentupa J. APS-I/APECED: the clinical disease and therapy. Endocrinol Metab Clin North Am 2002;31:295-320, vi.

51. Feingold KR, Elias PM. Endocrine-skin interactions. Cutaneous manifestations of pituitary disease, thyroid disease, calcium disorders, and diabetes. J Am Acad Dermatol 1987;17:921-40.

52. Feingold KR, Elias PM. Endocrine-skin interactions: Cutaneous manifestations of adrenal disease, pheochromocytomas, carcinoid syndrome, sex hormone excess and deficiency, polyglandular autoimmune syndromes, multiple endocrine neoplasia syndromes, and other miscellaneous disorde. J Am Acad Dermatol 1988;19:1-20.

53. Pappas PG, Kauffman CA, Andes D, et al. Clinical practice guidelines for the management of candidiasis: 2009 update by the Infectious Diseases Society of America. Clin Infect Dis 2009;48:503-35.

54. Rautemaa R, Richardson M, Pfaller MA, et al. Activity of amphotericin $\mathrm{B}$, anidulafungin, caspofungin, micafungin, posaconazole, and voriconazole against Candida albicans with decreased susceptibility to fluconazole from APECED patients on long-term azole treatment of chronic mucocutaneous candidiasis. Diagn Microbiol Infect Dis 2008;62:182-5. 